\title{
Cognitive outcome in adults with moderate disability after pneumococcal meningitis
}

\author{
Martijn Weisfelt ${ }^{\mathrm{a}, *}$, Diederik van de Beek ${ }^{\mathrm{a}}$, Martine Hoogman ${ }^{\mathrm{a}}$, \\ Clara Hardeman ${ }^{\mathrm{b}}$, Jan de Gans ${ }^{\mathrm{a}}$, Ben Schmand ${ }^{\mathrm{b}}$
}

\author{
${ }^{a}$ Department of Neurology, Academic Medical Center, P.O. Box 22660, 1100 DD Amsterdam, The Netherlands \\ ${ }^{\mathrm{b}}$ Department of Psychology, University of Amsterdam, Amsterdam, The Netherlands
}

Accepted 16 August 2005

Available online 10 October 2005

\section{KEYWORDS \\ Bacterial meningitis; Cognition disorders; Glasgow outcome scale; Disability evaluation; Streptococcus pneumoniae}

\begin{abstract}
Summary Objectives: To assess cognitive outcome and quality of life in patients with moderate disability after bacterial meningitis as compared to patients with good recovery.

Methods: Neuropsychological evaluation was performed in 40 adults after pneumococcal meningitis; 20 patients with moderate disability at discharge on the glasgow outcome scale (GOS score 4 ) and 20 with good recovery (GOS score 5 ).

Results: Patients with GOS score 4 had similar test results as compared to patients with GOS score 5 for the neuropsychological domains 'intelligence', 'memory' and 'attention and executive functioning'. Patients with GOS score 4 showed less cognitive slowness than patients with GOS score 5 . In a linear regression analysis cognitive speed was related to current intelligence, years of education and time since meningitis. Overall performance on the speed composite score correlated significantly with time since meningitis $(-0.62 ; P<0.001)$. Therefore, difference between both groups may have been related to a longer time between meningitis and testing for GOS four patients ( 29 vs. 12 months; $P<0.001$ ).

Conclusions: Patients with moderate disability after bacterial meningitis are not at higher risk for neuropsychological abnormalities than patients with good recovery. In addition, cognitive slowness after bacterial meningitis may be reversible in time.

(c) 2006 The British Infection Society. Published by Elsevier Ltd. All rights reserved.
\end{abstract}

\section{Introduction}

Bacterial meningitis in adulthood remains an important cause of mortality and long-term

* Corresponding author. Tel.: +3120566 3842; fax: + 3120 5669217.

E-mail address: m.weisfelt@amc.uva.nl (M. Weisfelt). neurological sequelae worldwide. ${ }^{1,2}$ Streptococcus pneumoniae and Neisseria meningitidis are the predominant cause of adulthood bacterial meningitis, with an overall case fatality rate of 15-33 and $9 \%$, respectively. ${ }^{1,2}$ Especially, meningitis due to S. pneumoniae is associated with considerable disability; hearing loss occurs in $22 \%$ of surviving patients. ${ }^{1,2}$ Outcome is frequently assessed by the glasgow outcome scale (GOS), which is a well 
validated scale with good interobserver agreement. ${ }^{2-4}$ The score on the GOS ranges from 1 to 5 : A score of 1 indicates death; 2, persistent vegetative state; 3 , severe disability; 4 , moderate disability (disabled but independent); 5, good recovery. ${ }^{4}$ Although GOS score 5 implies good recovery, cognitive impairment after meningitis in this outcome group occurs frequently. ${ }^{5}$ In a prospective study among 51 patients with good recovery after bacterial meningitis, one out of four patients after pneumococcal meningitis showed significant cognitive slowness, which was related with lower scores on general health and quality of life. ${ }^{5}$ Neuropsychological evaluation in the outcome group of moderately disability (GOS score 4 ) has not been performed. Assumingly, these patients have more severe disease and, therefore, are prone to neuropsychological impairment. However, a lower score on the GOS because of cranial nerve abnormalities, such as hearing loss, does not necessarily imply cognitive impairment. In this study we assessed cognitive outcome and quality of life in patients with moderate disability after bacterial meningitis as compared to patients with good recovery.

\section{Patients and methods}

This study was based on the Dutch Meningitis Cohort, a nationwide observational cohort study in the Netherlands. ${ }^{2}$ In this study 696 episodes of community-acquired acute bacterial meningitis, confirmed by cerebrospinal fluid cultures, were prospectively evaluated. Inclusion and exclusion criteria, treatment, laboratory results and outcome measures are described elsewhere. ${ }^{2}$ In summary, patients were eligible in this study if they were older than 16 years of age and were listed in the database of the Netherlands Reference Laboratory for Bacterial Meningitis from October 1998 to April 2002. This laboratory receives cerebrospinal fluid isolates from approx. $85 \%$ of all patients with bacterial meningitis in the Netherlands. ${ }^{2,6,7}$ The laboratory provided daily updates of the names of hospitals were patients with bacterial meningitis had been admitted 2-6 days earlier. The physicians, usually neurologists, were contacted and informed about the study. Subsequently, patients or their legal representative received written information concerning the study and were asked to give written informed consent; only participants from whom consent was obtained participated in the study. Information was collected by means of a case record form. All patients underwent a neurological examination at discharge, and outcomes were graded by use of the GOS.

\section{Neuropsychological evaluation}

We conducted a follow-up evaluation in adults surviving pneumococcal meningitis with moderate disability or good recovery on the GOS at discharge. Neuropsychological evaluation was done at least 6 months after discharge and patients eligible for this follow-up evaluation were patients aged 16-65 years with bacterial meningitis due to $S$. pneumoniae, who were discharged with GOS score 4 or 5 . Exclusion criteria were meningitis following head trauma or neurosurgical intervention, serious illnesses (other than meningitis), preexisting psychiatric disorders (including mental retardation) and insufficient mastery of Dutch language. Four cognitive domains were tested, as well as general health and quality of life. These domains were tested by the following test battery: (1) Intelligence, current intelligence was tested by the Groningen intelligence tests (GIT)-abbreviated version, consisting of subtests for verbal and visuospatial reasoning and numerical ability, ${ }^{8}$ premorbid intelligence was tested by the Dutch adult reading test; ${ }^{9}$ (2) memory, the Rey's auditory verbal learning test (AVLT) was used to test verbal memory, ${ }^{10}$ figural memory was tested by the subtest visual reproduction from the Wechsler memory scale revised (WMS$\mathrm{R}) ;^{10}$ (3) attention and executive functioning, Trailmaking test, Stroop colour-word test, category fluency, letter fluency, and the Wisconsin card sorting test (WCST); ${ }^{8,10}$ (4) reaction speed, simple and two-choice reaction time measured reaction speed; ${ }^{10}$ (5) questionnaires, determination of general health and quality of life were evaluated by means of the RAND-36 questionnaire, ${ }^{11,12}$ the profile of mood states (POMS) determined depressive mood disorders. ${ }^{10}$ The tests are described more extensively elsewhere. ${ }^{5}$

To explore the finding of differences in cognitive speed between outcome groups, we formed a speed composite score for each patient by calculation of mean Z-transformed values of tests in which cognitive speed is important (category and letter fluency, numerical speed, trailmaking $A$ and $B$, visual and binary choice reaction tasks); a higher score indicated worse performance. ${ }^{5}$

\section{Statistical methods}

Test results were analysed by multivariate analysis of variance for each neuropsychological domain separately with covariates age and education when 
applicable. For non-parametric testing, MannWhitney $U$, Chi-squared and Fisher's exact statistics were used; correlations are given by Pearson correlation coefficients.

\section{Results}

\section{Demographic and clinical data}

The characteristics of the Dutch meningitis cohort are described elsewhere. ${ }^{2}$ In summary, mean age was 50 years (SD 20) and 50\% was male. The causative organism was S. pneumoniae in 352 episodes (51\%), N. meningitidis in 257 (37\%), and 87 episodes $(12 \%)$ were due to other bacteria. Neurological examination at discharge was performed in all patients surviving pneumococcal meningitis: 175 patients $(50 \%)$ had GOS score $5 ; 50$ (14\%) GOS 4; 17 (5\%) GOS 3; 3 (1\%) GOS 2; and 107 patients $(30 \%)$ died (GOS 1$)$. A total of 220 patients were potentially eligible for cognitive testing; 55 consecutive patients were contacted for participation in this study. Forty patients were neuropsychological evaluated ( 20 with GOS score 4 and 20 with GOS score 5); three patients had insufficient mastery of Dutch language, six refused to participate and three patients had serious somatic illnesses. Demographic characteristics for patients eligible for testing $(n=220)$ and participants of this study $(n=40)$ were similar.

Clinical characteristics before admission and at presentation were similar for both outcome groups (Table 1), although the mean blood leucocyte count was higher for patients with GOS score $5(P=0.05)$. Overall, the classical triad of fever, headache and neck stiffness was present in 29 patients $(73 \%)$ and six patients $(15 \%)$ were comatose on admission. At discharge, patients with GOS score 4 had more neurological sequelae than patients with GOS score $5(P<0.001)$. Cranial nerve palsies (hearing impairment in all cases) were present in 14 patients (35\%) and cerebral focal deficits in five patients (13\%). Of patients with focal cerebral deficits, two had hemiparesis and three had severe ataxia (without hearing loss).

Demographic characteristics as gender, years of education and premorbid intelligence level were similar for both groups, although patients with GOS score 4 were older at the moment of testing than patients with GOS score 5 (Table 2). However, the mean time between discharge and neuropsychological evaluation was significantly longer for patients with GOS score 4 than for those with GOS score 5 ( 29 vs. 12 months; $P<0.001$ ).

Table 1 Baseline characteristics of patients with moderate disability (GOS score 4) and good recovery (GOS score 5) after pneumococcal meningitis

\begin{tabular}{|c|c|c|}
\hline Time point, parameter & GOS score $4(n=20)$ & GOS score $5(n=20)$ \\
\hline \multicolumn{3}{|l|}{ Baseline } \\
\hline Age at time of meningitis (years) & $51 \pm 8.2$ & $45 \pm 15.1$ \\
\hline Gender $(M: F)$ & $10: 10$ & $11: 9$ \\
\hline \multicolumn{3}{|l|}{ Before admission } \\
\hline Symptoms before admission $<24 \mathrm{~h}$ & $10 / 18$ & $10 / 20$ \\
\hline Seizures & $1 / 19$ & $1 / 20$ \\
\hline \multicolumn{3}{|l|}{ Presentation } \\
\hline Temperature $>38^{\circ} \mathrm{C}$, headache and neck stiffness & $13 / 20$ & $16 / 20$ \\
\hline Glasgow coma score $<8$ & $3 / 20$ & $3 / 20$ \\
\hline \multicolumn{3}{|l|}{ Laboratory } \\
\hline \multicolumn{3}{|l|}{ CSF leucocyte count (cells $/ \mathrm{mm}^{3}$ ) } \\
\hline$<100$ & $1 / 19$ & $0 / 20$ \\
\hline $100-999$ & $5 / 19$ & $4 / 20$ \\
\hline$\geq 1000$ & $13 / 19$ & $16 / 20$ \\
\hline Positive blood culture & $16 / 20$ & $18 / 20$ \\
\hline Blood leucocyte count $>10^{9}$ cells $/ \mathrm{l}$ & $16 / 20$ & $20 / 20^{a}$ \\
\hline \multicolumn{3}{|l|}{ Discharge } \\
\hline Cerebral focal deficits & $5 / 20$ & $0 / 20^{a}$ \\
\hline Cranial nerve palsies & $14 / 20$ & $0 / 20^{b}$ \\
\hline
\end{tabular}

\footnotetext{
Data are number/numbers evaluated, age is mean \pm SD.

a $P=0.05$ (Fisher's exact test, two-tailed).

b $P<0.001$ (Fisher's exact test, two-tailed).
} 


\section{Neuropsychological data}

Multivariate analyses of covariance within each neuropsychological domain showed significant overall group differences for 'reaction speed' (Pillais' trace; $P=0.02$ ), but not for 'intelligence' $(P=0.16)$, 'memory' $(P=0.60)$ and 'attention and executive functioning' $(P=0.09)$. Within the domain of reaction speed, patients with GOS score 4 had superior scores on the binary choice reaction task, compared with patients with GOS score 5 (Table 2).

Patients with GOS score 4 had significant lower scores on the speed composite score (indicating better performance) than patients with GOS score 5 (univariate test with covariates age and years of education; $P=0.009$ ). However, in a linear regression analysis the speed composite score was significantly associated with current intelligence (IQ; $P=0.03)$, education $(P=0.05)$ and time since, meningitis $(P=0.05)$; associations with age and GOS score were no longer significant. Overall performance of patients on the speed composite score correlated significantly with time since meningitis $(-0.62 ; P<0.001)$, with covariates age, IQ and education.

To evaluate the clinical relevance of test results we defined a test score as clinically abnormal if below one standard deviation from the mean of a control group derived from a previous study. ${ }^{5}$ There was a considerable heterogeneity between test results within the group of patients with GOS score 4. For patients with cranial nerve palsies at discharge the mean number of abnormal test results was significantly lower compared to patients with cerebral focal deficits (6 vs. $2 ; P=0.001$ ), and

Table 2 Demographic characteristics and test scores in neuropsychological evaluation of patients with moderate disability (GOS score 4) and good recovery (GOS score 5) after pneumococcal meningitis

\begin{tabular}{|c|c|c|c|}
\hline Time point, parameter & GOS score $4(n=20)$ & GOS score $5(n=20)$ & $P$ value $^{a}$ \\
\hline \multicolumn{4}{|l|}{ Baseline } \\
\hline Age at time of testing (years) & $53.6 \pm 8.1$ & $45.8 \pm 15.2$ & 0.05 \\
\hline Education (years) & $12.7 \pm 1.3$ & $13.4 \pm 3.6$ & 0.46 \\
\hline Time from discharge to testing (months) & $29.3 \pm 12.7$ & $12.2 \pm 4.7$ & $<0.001$ \\
\hline Premorbid intelligence (IQ) & $98.9 \pm 15.6$ & $94.5 \pm 17.8$ & 0.41 \\
\hline \multicolumn{4}{|l|}{ Intelligence (GIT) } \\
\hline Intelligence (IQ) & $110.3 \pm 18.9$ & $104.8 \pm 15.4$ & 0.32 \\
\hline Verbal reasoning $(\mathrm{T} \text {-score })^{\mathrm{b}}$ & $58.1 \pm 9.3$ & $58.1 \pm 8.1$ & 1.00 \\
\hline Visuospatial reasoning (T-score) & $53.6 \pm 9.9$ & $52.4 \pm 10.9$ & 0.72 \\
\hline Numerical speed (T-score) & $53.2 \pm 10.3$ & $46.3 \pm 9.6$ & 0.04 \\
\hline \multicolumn{4}{|l|}{ Memory } \\
\hline Rey AVLT immediate (raw) & $41.7 \pm 8.7$ & $44.1 \pm 9.1$ & 0.39 \\
\hline Rey AVLT delayed (raw) & $8.5 \pm 3.3$ & $9.4 \pm 3.2$ & 0.42 \\
\hline Rey AVLT recognition (raw) & $28.5 \pm 2.7$ & $28.8 \pm 1.4$ & 0.60 \\
\hline WMS-R immediate (T-score) & $59.5 \pm 11.8$ & $51.6 \pm 11.5$ & 0.04 \\
\hline WMS-R delayed (T-score) & $56.8 \pm 13.5$ & $50.8 \pm 10.5$ & 0.12 \\
\hline \multicolumn{4}{|l|}{ Attention and executive functioning } \\
\hline Trailmaking A (T-score) & $54.1 \pm 12.1$ & $49.4 \pm 12.6$ & 0.05 \\
\hline Trailmaking B (T-score) & $53.8 \pm 9.7$ & $46.7 \pm 9.9$ & 0.03 \\
\hline Stroop colour-word test (T-score) & $54.9 \pm 10.4$ & $49.4 \pm 12.6$ & 0.14 \\
\hline Category fluency (T-score) & $54.2 \pm 8.6$ & $51.9 \pm 7.9$ & 0.38 \\
\hline Letter fluency (T-score) & $50.1 \pm 11.5$ & $43.0 \pm 9.1$ & 0.04 \\
\hline No. WCST categories (raw) & $4.6 \pm 1.3$ & $4.6 \pm 1.7$ & 0.94 \\
\hline No. WCST total errors (raw) & $42.9 \pm 17.8$ & $31.3 \pm 18.4$ & 0.06 \\
\hline No. WCST perseverative errors (raw) & $23.6 \pm 12.6$ & $14.5 \pm 9.6$ & 0.02 \\
\hline \multicolumn{4}{|l|}{ Reaction speed } \\
\hline Visual reaction task, dominant hand (ms) & $280.3 \pm 75.4$ & $301.0 \pm 60.5$ & 0.41 \\
\hline Visual reaction task, non-dominant hand (ms) & $273.3 \pm 48.7$ & $305.5 \pm 65.8$ & 0.14 \\
\hline Binary choice reaction task (ms) & $445.3 \pm 113.2$ & $559.7 \pm 143.6$ & 0.02 \\
\hline
\end{tabular}


similar to GOS five patients and controls. Scores on the speed composite score were similar for patients with and without cerebral deficits at discharge.

Scores on general health and quality of life (RAND-36) revealed lower scores on the item 'physical functioning' for patients with GOS score 4 compared with those with GOS score 5 (Table 3). On other items no significant differences were found. Patients with cerebral focal deficits had lower scores on the item 'physical functioning' compared with patients without cerebral deficits (score on item 55 vs. 78; $P=0.02$ ). In addition, scores on the item 'physical functioning' correlated significantly with the speed composite score $(-0.35 ; P=0.03)$. No differences were found on the depression scale (POMS) for both outcome groups and there was no significant correlation between the item 'depression' on the POMS and the results on the general speed composite score.

\section{Discussion}

We found that adults with moderate disability after pneumococcal meningitis are not at higher risk for cognitive impairment than patients with good recovery. Cognitive impairment occurs frequently after bacterial meningitis. Other studies on cognitive impairment among adults after bacterial meningitis described cognitive slowness, impairment of psychomotor performance, reduction in visuoconstructive performance and higher scores on depression scales. ${ }^{5,13-16}$ In a previous study we found that even adults with good recovery after pneumococcal meningitis are at significant risk for cognitive slowness. ${ }^{5}$ In this study, 51 patients with good recovery after meningococcal and pneumococcal meningitis were examined. One out of four patients with good recovery after pneumococcal meningitis had significant cognitive impairment, mainly consisting of cognitive slowness. Patients after meningococcal meningitis had comparable test scores as controls. ${ }^{5}$ In the present study, patients with moderate disability due to focal cerebral deficits were at higher risk for cognitive impairment than patients without these sequelae.

Cognitive speed was related to time since meningitis. In a linear regression model cognitive speed was related to current intelligence, years of education and time since meningitis. Although patients with moderate disability were significantly faster in time dependent tests than those with GOS score 5 , both groups had similar test scores after correction for these variables. Although the difference in time interval since meningitis is a considerable shortcoming of our study, the relation between cognitive slowness and time suggests that cognitive slowness in adults after pneumococcal meningitis may be in part reversible. The dynamic character of sequelae in survivors of bacterial meningitis has been described previously and implies that time since illness is an important factor in follow-up studies of adults after pneumococcal meningitis. ${ }^{14,17}$ Our finding is in agreement with results of a prospective longitudinal cohort study on long-term cognitive functioning in children after bacterial meningitis. ${ }^{18}$ This study showed a significant 'catch-up' of children with cognitive impairment after meningitis in executive function and cognitive speed, which is largely comparable with our results.

Besides the difference in time between discharge and neuropsychological evaluation there are several other shortcomings in this study. The number of patients that are neuropsychological evaluated is small, which impaired statistical correction for the number of tests. However, demographic features for the participants and the eligible patients were similar and results were compared to a control group that was similar to features as age and education. Nevertheless, our results should be

Table 3 Scores on general health and quality of life (RAND-36) for adults with moderate disability (GOS score 4) and good recovery (GOS score 5) after pneumococcal meningitis

\begin{tabular}{llll}
\hline Characteristic & GOS score $4(n=20)$ & GOS score 5 $(n=20)$ & $P$ value \\
\hline Physical functioning & $68.2 \pm 22.1$ & $81.1 \pm 15.9$ & 0.05 \\
Social functioning & $69.1 \pm 29.0$ & $81.3 \pm 24.5$ & 0.16 \\
Role impairment due to physical problems & $65.8 \pm 44.3$ & $60.0 \pm 42.5$ & 0.68 \\
Role impairment due to emotional problems & $64.9 \pm 40.8$ & $76.7 \pm 39.1$ & 0.36 \\
Mental health & $73.7 \pm 15.7$ & $78.0 \pm 12.9$ & 0.35 \\
Vitality & $57.1 \pm 17.1$ & $57.0 \pm 21.3$ & 0.99 \\
Pain & $73.8 \pm 27.7$ & $85.4 \pm 18.4$ & 0.15 \\
Experienced general health & $66.3 \pm 15.5$ & $66.0 \pm 13.2$ & 0.95
\end{tabular}

Data are mean $\pm S D$. RAND-36; higher score indicates better performance.

a Two-tailed $P$ values ( $t$-test); GOS 4 vs. GOS 5 patients. 
confirmed in a study with larger number of adults with pneumococcal meningitis. Furthermore, mood disorders are often present after meningitis and are a potential confounder of cognitive test results. ${ }^{5}$ There was no significant correlation between depressed mood and cognitive speed and scores on the POMS depression-subscale were similar for both outcome groups. Therefore, it is unlikely that our results were confounded by depression.

Research on cognitive outcome in bacterial meningitis is scarce and often with methodological shortcomings. However, this follow-up evaluation is designed for testing of multiple cognitive domains and the population is relatively homogenous. Our finding that loss of cognitive speed in adults after pneumococcal meningitis may be reversible in time is important information for patients presenting with cognitive complaints after bacterial meningitis. The association between the severity of neurological sequelae after pneumococcal meningitis and neuropsychological abnormalities is weak. ${ }^{14}$ Since the categories of the GOS are very broad, the GOS seems to be less adequate as an instrument to evaluate cognitive outcome after adulthood pneumococcal meningitis. Classification of patients based on the nature of their neurological sequelae, such as cranial-nerve palsies vs. cerebral focal abnormalities may be more useful to identify patients who are at risk for cognitive impairments.

Bacterial meningitis is a complex disorder in which neurological injury is caused in part by the causative organism and in part by the host's own inflammatory response. Recently, the European dexamethasone in adulthood bacterial meningitis study showed that adjunctive dexamethasone therapy reduces both mortality and morbidity in adults with bacterial meningitis. ${ }^{3}$ Ever since, adjunctive dexamethasone is routine therapy for most adults with suspected bacterial meningitis. ${ }^{19-21}$ In experimental meningitis, dexamethasone as an adjunct to antibiotic treatment aggravates neuronal damage in the hippocampal formation ${ }^{22}$ and may potentiate ischemic injury to neurons, ${ }^{23}$ so it is important to assess adverse effects-e.g. whether steroids may prevent death but worsen cerebral cortical functioning. ${ }^{20}$ Therefore, further neuropsychological evaluation is needed of patients with disability and good recovery after bacterial meningitis, treated with and without adjunctive dexamethasone therapy.

\section{Acknowledgements}

Financial support: This study was supported by a grant from the Meningitis Research Foundation, UK.
Conflict of interest: There is no potential conflict of interest for any of the authors involved.

\section{References}

1. Durand ML, Calderwood SB, Weber DJ, Miller SI, Southwick FS, Caviness VS Jr, et al. Acute bacterial meningitis in adults. A review of 493 episodes. N Engl J Med 1993;328(1):21-8.

2. van de Beek $D$, de Gans J, Spanjaard L, Weisfelt $M$, Reitsma JB, Vermeulen M. Clinical features and prognostic factors in adults with bacterial meningitis. $N$ Engl $J$ Med 2004;351(18):1849-59.

3. de Gans J, van de Beek D. Dexamethasone in adults with bacterial meningitis. N Engl J Med 2002;347(20):1549-56.

4. Jennet B, Teasdale G. Management of head injuries. Philadelphia: Davies; 1981.

5. van de Beek D, Schmand B, de Gans J, Weisfelt M, Vaessen $\mathrm{H}$, , Dankert J, et al. Cognitive impairment in adults with good recovery after bacterial meningitis. $J$ Infect Dis 2002;186(7):1047-52.

6. Netherlands Reference Laboratory for bacterial meningitis (AMC/RIVM). Bacterial meningitis in The Netherlands: Annual report 1999. University of Amsterdam; 2000.

7. van de Beek D, de Gans J, Spanjaard L, Vermeulen M, Dankert J. Antibiotic guidelines and antibiotic use in adult bacterial meningitis in The Netherlands. J Antimicrob Chemother 2002;49(4):661-6.

8. Luteijn F, van der Ploeg F. Groningen intelligentie test. Amsterdam: Swets and Zeitlinger; 1983.

9. Schmand B, Lindeboom J, van Harskamp F. De Nederlandse leestest voor volwassenen. Amsterdam: Swets and Zeitlinger; 1992.

10. Lezak MD, Howieson BD, Loring DW. Neuropsychological assessment. 4th ed. Oxford: Oxford University Press; 2004.

11. Brazier JE, Harper R, Jones NM, O'Cathain A, Thomas KJ, Usherwood $\mathrm{T}$, et al. Validating the SF-36 health survey questionnaire: New outcome measure for primary care. BMJ 1992;305(6846):160-4.

12. van der Zee $\mathrm{K}$, Sanderman R. Het meten van de algemene gezondheidstoestand met de RAND-36. Groningen: Groningen University; 1993.

13. Merkelbach S, Sittinger H, Schweizer I, Muller M. Cognitive outcome after bacterial meningitis. Acta Neurol Scand 2000; 102(2):118-23.

14. Bohr V, Rasmussen N, Hansen B, Gade A, Kjersem H, Johnsen N, et al. Pneumococcal meningitis: An evaluation of prognostic factors in 164 cases based on mortality and on a study of lasting sequelae. J Infect 1985;10(2):143-57.

15. Naess A, Halstensen A, Nyland H, Pedersen SH, Moller P, Borgmann $\mathrm{R}$, et al. Sequelae one year after meningococcal disease. Acta Neurol Scand 1994;89(2):139-42.

16. Zahner B, Harrer M, Erbguth F, Stefan H, Neundorfer B. Purulent meningoencephalitis-studies of disease progression and prognosis. Fortschr Neurol Psychiatr 1995; 63(6):220-6.

17. Bruyn GA, Kremer HP, de Marie S, Padberg GW, Hermans J, van Furth R. Clinical evaluation of pneumococcal meningitis in adults over a twelve-year period. Eur J Clin Microbiol Infect Dis 1989;8(8):695-700. 
18. Anderson V, Anderson P, Grimwood K, Nolan T. Cognitive and executive function 12 years after childhood bacterial meningitis: Effect of acute neurologic complications and age of onset. J Pediatr Psychol 2004;29(2):67-81.

19. Tunkel AR, Hartman BJ, Kaplan SL, Kaufman BA, Roos KL, Scheld WM, et al. Practice guidelines for the management of bacterial meningitis. Clin Infect Dis 2004;39(9):1267-84.

20. van de Beek D, de Gans J, McIntyre P, Prasad K. Steroids in adults with acute bacterial meningitis: A systematic review. Lancet Infect Dis 2004;4(3):139-43.
21. Chaudhuri A. Adjunctive dexamethasone treatment in acute bacterial meningitis. Lancet Neurol 2004;3(1): 54-62.

22. Leib SL, Heimgartner C, Bifrare YD, Loeffler JM, Taauber MG. Dexamethasone aggravates hippocampal apoptosis and learning deficiency in pneumococcal meningitis in infant rats. Pediatr Res 2003;54(3):353-7.

23. Sapolsky RM, Pulsinelli WA. Glucocorticoids potentiate ischemic injury to neurons: Therapeutic implications. Science 1985;229(4720):1397-400. 